\title{
Gamificação no Ensino Superior em Ciência da Computação: Uma Revisão Sistemática da Literatura
}

Felipe Augusto Feichas, Universidade Federal de Itajubá, feichas2000@gmail.com, https://orcid.org/0000-0001-5062-9833

Rodrigo Duarte Seabra, Universidade Federal de Itajubá, rodrigo@unifei.edu.br, https://orcid.org/0000-0002-7465-2963

Adler Diniz de Souza, Universidade Federal de Itajubá, adlerunifei@gmail.com, https://orcid.org/0000-0002-9393-398X

Resumo: Gamificação é a prática de aplicar elementos e princípios de jogos em ambientes não relacionados a jogos. Esse recurso tem sido amplamente utilizado no ensino superior em diferentes áreas do conhecimento, incorporando elementos de $e$ learning com gamificação, com o objetivo de melhorar a qualidade do ensino. Esta pesquisa, por meio de uma revisão sistemática da literatura, discutirá a eficácia da gamificação, seus benefícios e como ela vem sendo aplicada no âmbito da Ciência da Computação. A partir da revisão realizada foi verificado que a maioria das publicações apresentou resultados positivos com a gamificação, principalmente no engajamento e na motivação dos estudantes. Contudo, em alguns casos, foi identificada uma diminuição dos benefícios em longo prazo, dependente da forma como a prática é aplicada.

Palavras-chave: gamificação, ensino superior, ciência da computação, revisão sistemática da literatura.

\section{Gamification in Higher Education in Computer Science: A Systematic Literature Review}

\begin{abstract}
Gamification is the practice of applying elements and principles of games to non-games environments. This resource has been widely used in higher education in different areas of knowledge, combining elements of e-learning with gamification, to improve the quality of teaching. This research, through a systematic literature review, will discuss the effectiveness of gamification, its benefits and how it has been applied to the scope of Computer Science. From the review carried out, we found that most publications showed positive results with gamification, mainly as regards student engagement and motivation. However, in some cases, a decrease in long-term benefits has been identified, depending on how the practice is applied.
\end{abstract}

Keywords: gamification, higher education, computer science, systematic literature review.

\section{INTRODUÇÃO}

Para muitos estudantes, o método tradicional de ensino é percebido como ineficaz e desestimulador. Embora professores, em geral, busquem por novas abordagens instrucionais, manter os estudantes engajados e evitar a evasão é um dos grandes desafios a serem alcançados (LETHTONEN et al., 2015). O uso de elementos de jogos como ferramenta de aprendizagem é uma alternativa que possui um poder motivacional notável de incentivar os alunos a se envolverem com o processo de aprendizado (TSAY et al., 2018; MACENA et al., 2020). Nesse contexto, a gamificação surge como uma nova abordagem no cenário educacional, estimulando a 
obtenção do conhecimento e, por conseguinte, o aumento do engajamento $\mathrm{e}$ desempenho dos estudantes, se comparada ao método tradicional de ensino. Na prática, a gamificação utiliza elementos de jogos em ambientes não relacionados a jogos, permitindo aos estudantes vencerem desafios e melhorar a sua aprendizagem (SPENCE et al., 2012). Apesar de existirem revisões sistemáticas da literatura (RSL) direcionadas à aplicação da gamificação na educação (NAH et al., 2014; DICHEVA et al., 2015), este estudo se difere dos demais por apresentar uma revisão focada no uso da gamificação no ensino superior em Ciência da Computação. Este trabalho, portanto, apresenta uma RSL sobre o uso da gamificação no aprendizado de disciplinas da Ciência da Computação, objetivando discutir sua eficácia no processo de aprendizagem. O estudo visa contribuir com futuros pesquisadores, docentes e discentes na busca por práticas efetivas da gamificação no processo de aprendizagem em Ciência da Computação, sendo esta sua principal contribuição. $O$ artigo está estruturado da seguinte forma: a Seção 2 apresenta a metodologia de pesquisa utilizada, assim como as questões de pesquisa, as etapas e os processos executados. A Seção 3 apresenta os resultados do estudo. Na Seção 4 é tecida uma discussão sobre as questões de pesquisa definidas na Seção 2. Finalmente, a Seção 5 apresenta as considerações finais do estudo.

\section{DEFINIÇÃO DA PESQUISA}

O propósito desta RSL foi investigar o uso da gamificação no processo de aprendizado, do ponto de vista dos pesquisadores, no contexto do ensino superior em Ciência da Computação. Esta pesquisa se justifica, uma vez que explora a literatura em busca da eficácia da gamificação no processo de aprendizagem. Ademais, apresenta os principais benefícios obtidos com a gamificação e a forma como ela vem sendo implementada em sala de aula. A metodologia utilizada na pesquisa foi baseada em Kitchenham e Charters (2007) e abordará as questões de interesse, o protocolo de busca e seleção dos repositórios, além da execução, aplicação dos critérios de inclusão e exclusão e extração dos dados.

\subsection{Questões de Interesse}

As questões de interesse têm como propósito principal fornecer uma visão sobre a eficácia da gamificação em disciplinas da área de Ciência da Computação. Para tanto, foram definidas três questões de pesquisa: Q1: Que evidências existem para a eficácia da gamificação em disciplinas da Computação no ensino superior? A principal questão consiste em coletar informações sobre os resultados dos estudos publicados e as evidências relatadas com a aplicação da gamificação nesse contexto. Q2: Quais os benefícios obtidos no processo de aprendizagem com a gamificação? A partir da questão anterior buscou-se identificar os benefícios que os pesquisadores obtiveram com o uso da gamificação. Q3: Como a gamificação está sendo aplicada em disciplinas da Computação no ensino superior? A questão visa investigar como a gamificação vem sendo aplicada e as principais ferramentas empregadas.

\subsection{Protocolo de Busca e Seleção}

Para delinear o escopo da pesquisa foram estabelecidos alguns critérios para garantir a confiabilidade e a viabilidade da execução da revisão, permitindo o acesso aos dados e a abrangência do estudo. A pesquisa foi realizada a partir de três repositórios digitais - Scopus, IEEE e ScienceDirect - por meio dos seus respectivos mecanismos de 
busca. Os critérios para a seleção dos repositórios foram os seguintes: (i) conter uma grande quantidade de publicações relacionadas à Ciência da Computação; (ii) permitir o uso de expressões lógicas ou recurso equivalente nos mecanismos de busca; (iii) serem repositórios reconhecidos do ponto de vista acadêmico.

A pesquisa está restrita às publicações obtidas, exclusivamente, a partir dos repositórios selecionados e o período considerado das publicações foi de 01 de janeiro de 2015 até 20 de junho de 2020. A data inicial foi escolhida de modo a obter as publicações mais recentes sobre o tema. A data final foi estabelecida a partir da data de início deste estudo. Para a realização da pesquisa foi selecionado o idioma inglês, devido ao fato de ser adotado pela grande maioria das conferências e periódicos internacionais. As palavras-chave utilizadas nas buscas foram: gamification, gamified, gamify, gameful, computer science, learning, e-learning, teach, teaching, university, graduation, undergraduate, university graduate, higher education e faculty. Como cada repositório possui suas peculiaridades e especificações, a expressão de busca variou para cada um. O processo de seleção ocorreu em três fases, a saber: a primeira fase envolveu a catalogação dos dados coletados e a exclusão de falsos positivos, ou seja, remoção das publicações com temas que não se relacionavam ao objetivo da pesquisa. $\mathrm{Na}$ segunda fase, foram lidos os resumos e aplicados critérios de exclusão (CE) e critérios de inclusão (CI). Na terceira fase, foram lidos os textos completos, aplicados os CE e CI, e extraídos os conceitos de gamificação abordados. Os CI podem ser vistos no Quadro 1 e os CE podem ser consultados no Quadro 2. O catálogo final contém o título, o ano da publicação, os autores e o percentil Scopus (Quadro 4).

Quadro 1 - Conjunto de critérios de inclusão (CI). Fonte: Os autores.

\begin{tabular}{|c|l|}
\hline Critério & \multicolumn{1}{|c|}{ Descrição } \\
\hline CI-01 & Publicações que mencionam a gamificação (questão Q1). \\
\hline CI-02 & Publicações que discutem os benefícios da gamificação (questão Q2). \\
\hline CI-03 & Publicações que descrevem a aplicação da gamificação (questão Q3). \\
\hline CI-04 & Publicações que possuam evidênnis empíricas. \\
\hline CI-05 & Publicações aplicadas no ensino superior em ciência da computação. \\
\hline CI-06 & Publicações no idioma inglês. \\
\hline
\end{tabular}

Quadro 2 - Conjunto de critérios de exclusão (CE). Fonte: Os autores.

\begin{tabular}{|c|l|}
\hline Critério & \multicolumn{1}{c|}{ Descrição } \\
\hline CE-01 & Não serão selecionadas publicações nas quais as palavras-chave e suas variações não estão presentes. \\
\hline CE-02 & Não serão selecionadas publicações que não respondam a pelo menos uma das questões Q1, Q2 e Q3. \\
\hline CE-03 & Não serão selecionadas publicações de áreas diferentes da ciência da computação. \\
\hline CE-04 & Não serão selecionadas publicações aplicadas em níveis diferentes do ensino superior. \\
\hline CE-05 & $\begin{array}{l}\text { Não serão selecionadas publicações que descrevam e/ou apresentem pré-pesquisas, design de game, } \\
\text { trabalhos incompletos, tutoriais, cursos, workshops e similares. }\end{array}$ \\
\hline CE-06 & Não serão selecionadas publicações como documentos técnicos e RSL ou mapeamento da literatura. \\
\hline CE-07 & Não serão selecionadas publicações que não tenham versão escrita em inglês. \\
\hline CE-08 & Não serão aceitas publicações duplicadas. \\
\hline CE-09 & Não serão aceitas publicações que não estejam disponíveis por completo para download. \\
\hline CE-10 & Não serão aceitas publicações de percentil Scopus menor que 50. \\
\hline
\end{tabular}

\subsection{Execução da Pesquisa}

A expressão de busca foi definida da seguinte maneira: inicialmente, foi realizada uma busca manual nas primeiras publicações relacionadas à gamificação. Após a definição da expressão de busca do Quadro 3, foi iniciada a extração das publicações e a estratégia adotada foi o processo de snowballing retroativo. 
Quadro 3 - Expressões de busca nos repositórios. Fonte: Os autores.

\begin{tabular}{|c|l|}
\hline Repositório & \multicolumn{1}{c|}{ Expressão de Busca } \\
\hline \multirow{5}{*}{ Scopus } & $\begin{array}{l}\text { (KEY (gamification) OR KEY (gamified) OR KEY (gamify) OR KEY (gameful) OR TITLE } \\
\text { (gamification) OR TITLE (gamified) OR TITLE (gamify) OR TITLE (gameful)) AND TITLE- } \\
\text { ABS-KEY (learning OR teach OR teaching OR educate OR e-learning OR educational OR course } \\
\text { OR education) AND TITLE-ABS-KEY (university graduate OR graduation OR university OR } \\
\text { higher education OR faculty OR undergraduate) AND TITLE-ABS-KEY (computer science OR } \\
\text { computer science course OR introductory computer science) }\end{array}$ \\
\hline \multirow{5}{*}{ IEEE } & $\begin{array}{l}\text { (Author Keywords: gamification OR Document Title: gamification OR Abstract: gamification OR } \\
\text { Author Keywords: gamified OR Document Title: gamified OR Abstract: gamified OR Author } \\
\text { Keywords: gamify OR Document Title: gamify OR Abstract: gamify OR Author Keywords: } \\
\text { gameful OR Document Title: gameful OR Abstract: gameful) AND (learning OR teach OR } \\
\text { teaching OR educate OR e-learning OR educational OR course OR education) AND (university } \\
\text { OR higher education OR faculty OR graduation OR university graduate OR undergraduate) AND } \\
\text { (computer science OR computer science course OR introductory computer science) }\end{array}$ \\
\hline Direct & $\begin{array}{l}\text { (gamification OR gamified OR gamify OR gameful) AND (learning OR teach OR teaching OR e- } \\
\text { learning OR course OR education) AND (university OR higher education OR graduation OR } \\
\text { university) AND (computer science) }\end{array}$ \\
\hline
\end{tabular}

A busca realizada com essas expressões retornou um total de 151 publicações, distribuídas entre os repositórios da seguinte maneira: Scopus - 50; IEEE - 76; Science Direct - 25. Como nem todos os documentos encontrados estavam alinhados com a questão de pesquisa, essas publicações foram submetidas a uma triagem (Figura 1) para que somente os artigos relevantes, para o propósito do estudo, fossem utilizados. O link a seguir contém a relação de publicações encontradas nos respectivos repositórios, bem como os critérios que justificaram sua inclusão ou exclusão desse estudo. https://docs.google.com/spreadsheets/d/1VPvZov3vPCfVfvjc1_DsxXsk75QaM4ptMU EQGu8eRLM/edit?usp=sharing.

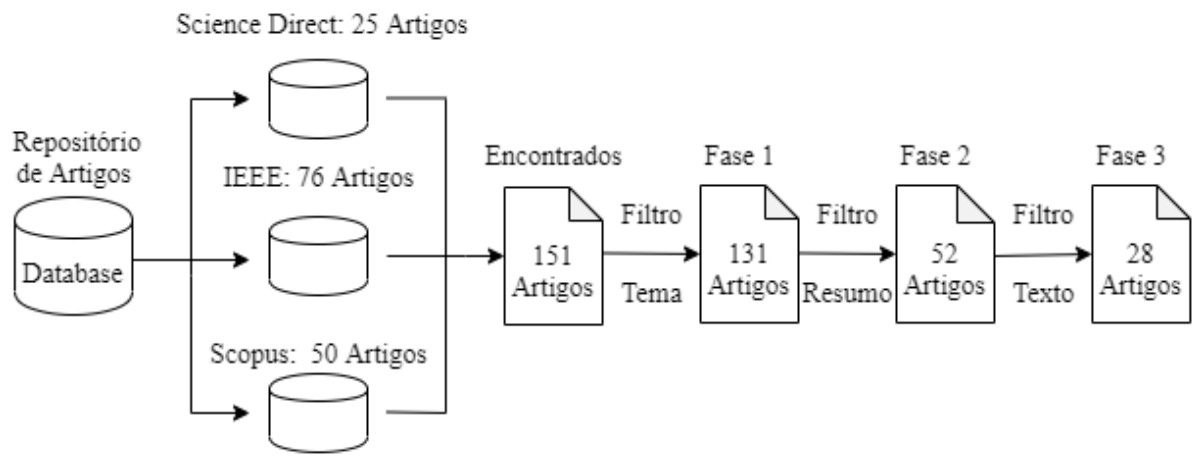

Figura 1 - Metodologia de busca da revisão sistemática. Fonte: Os autores.

$\mathrm{Na}$ primeira fase, foram removidas as publicações com temas que não se relacionavam ao objetivo da pesquisa, e o catálogo foi reduzido para 131 publicações. $\mathrm{Na}$ segunda fase, foi realizada a leitura de todos os resumos das publicações, sendo aplicados os CE, removendo do levantamento 79 artigos. Para a terceira fase, foram selecionadas 52 publicações. Nesta última fase, para cada uma das publicações restantes foi realizada a leitura completa do texto. Com os critérios aplicados, foram removidas 24 publicações, sendo selecionadas 28 para serem analisadas e discutidas neste trabalho. As publicações selecionadas foram tabuladas no Quadro 4, que contém as principais informações extraídas de cada trabalho: título, ano da publicação, autores e percentil, estando todas elas disponíveis no documento de controle. 
Quadro 4 - Catálogo de publicações. Fonte: Os autores.

\begin{tabular}{|c|c|c|c|c|}
\hline ID & Título & Ano & Autores & Percentil \\
\hline $\mathbf{P 1}$ & $\begin{array}{l}\text { The Impact of Gamification on Learning Outcomes of Computer } \\
\text { Science Majors }\end{array}$ & 2020 & Ahmad, A. et al. & 99th \\
\hline $\mathbf{P 2}$ & $\begin{array}{l}\text { Teaching parallelism with gamification in cellular automaton } \\
\text { environments }\end{array}$ & 2020 & $\begin{array}{l}\text { Hardasmal, A. J. T.; } \\
\text { Salguero, A. G. }\end{array}$ & 99th \\
\hline $\mathbf{P 3}$ & Gamification and computer science students' activity & 2020 & García-Iruela, M. et al. & 99th \\
\hline $\mathbf{P 4}$ & $\begin{array}{l}\text { Analyzing the impact of leaderboards in introductory programming } \\
\text { courses short-length activities }\end{array}$ & 2019 & Pontes, R. G. et al. & 99th \\
\hline $\mathbf{P 5}$ & $\begin{array}{l}\text { Implementing gamification in a university-level UML modeling } \\
\text { course: a case study }\end{array}$ & 2019 & Jurgelaitis, M. et al. & 99th \\
\hline P6 & Motivating students beyond course requirements with a serious game & 2019 & Watson, S.; Lipford, R. & 99th \\
\hline P7 & $\begin{array}{l}\text { Smile goes gaming: gamification in a classroom response system for } \\
\text { academic teaching }\end{array}$ & 2019 & Feldbusch, L. et al. & 99th \\
\hline P8 & $\begin{array}{l}\text { Achievements and usage of learning materials in computer science } \\
\text { hybrid courses }\end{array}$ & 2019 & Bernik, A. et al. & 99th \\
\hline P9 & $\begin{array}{l}\text { Gamification and evaluation of the use the agile tests in software } \\
\text { quality subjects: the application of case studies }\end{array}$ & 2018 & $\begin{array}{l}\text { Elgrably, I.S.; Oliveira, } \\
\text { S. R. B }\end{array}$ & 74th \\
\hline P10 & $\begin{array}{l}\text { Playing and learning with gamification: an in-class concurrent and } \\
\text { distributed programming activity }\end{array}$ & 2015 & $\begin{array}{l}\text { Maia, R.F.; Graeml, } \\
\text { F.R. }\end{array}$ & 59 th \\
\hline P11 & $\begin{array}{l}\text { An empirical study inspecting the benefits of gamification applied to } \\
\text { university classes }\end{array}$ & 2015 & Fracz, W. & 99th \\
\hline P12 & $\begin{array}{l}\text { Implementing gamification techniques into university study path - a } \\
\text { case study }\end{array}$ & 2015 & Laskowski, M. & 99th \\
\hline P13 & $\begin{array}{l}\text { The effect of achievement badges on students' behavior: an empirical } \\
\text { study in a university-level computer science course }\end{array}$ & 2015 & Hakulinen, L. et al. & 99th \\
\hline P14 & $\begin{array}{l}\text { Increasing engagement in a network security management course } \\
\text { through gamification }\end{array}$ & 2019 & Martin, S. et al. & 99th \\
\hline P15 & $\begin{array}{l}\text { Toward improving collaborative behaviour during competitive } \\
\text { programming assignments }\end{array}$ & 2019 & $\begin{array}{l}\text { Gonzalez-Escribano, A. } \\
\text { et al. }\end{array}$ & 99th \\
\hline P16 & $\begin{array}{l}\text { Studying the impact of gamification on learning and engagement of } \\
\text { introverted and extroverted students }\end{array}$ & 2019 & Smiderle, R. et al. & 99th \\
\hline P17 & $\begin{array}{l}\text { 2TSW: Automated assessment of computer programming } \\
\text { assignments in a gamified web based system }\end{array}$ & 2019 & Giuseppina, P. et al. & 87 th \\
\hline P18 & Gamification in software engineering education & 2019 & Ivanova, G. et al. & 99th \\
\hline P19 & $\begin{array}{l}\text { Digital badges for motivating introductory programmers: qualitative } \\
\text { findings from focus groups }\end{array}$ & 2018 & Facey-Shaw, L. et al. & 99th \\
\hline P20 & $\begin{array}{l}\text { A model for gamifying programming education: university-level } \\
\text { programming course quantified }\end{array}$ & 2018 & Kaila, E. et al. & 97th \\
\hline P21 & Gamification technique for teaching programming & 2018 & Carreno-Leon, M. et al. & 99th \\
\hline $\mathbf{P 2 2}$ & A gamified approach to achieve excellence in programming & 2018 & Kumar, B.; Sharma, K. & 99th \\
\hline $\mathbf{P 2 3}$ & Introducing gamification into e-learning university courses & 2017 & Bernik, A. et al. & 99th \\
\hline $\mathbf{P 2 4}$ & $\begin{array}{l}\text { Gamifying the learning of design patterns in software engineering } \\
\text { education }\end{array}$ & 2016 & Bartel, A.; Hagel, G. & 99th \\
\hline $\mathbf{P 2 5}$ & Enhancing the CS1 student experience with gamification & 2015 & Sprint, G. and Cook, D. & 99th \\
\hline $\mathbf{P 2 6}$ & $\begin{array}{l}\text { Enhancing student learning experience with technology-mediated } \\
\text { gamification: an empirical study }\end{array}$ & 2018 & Tsay, C. et al. & 99th \\
\hline $\mathbf{P 2 7}$ & $\begin{array}{l}\text { To gamify or not to gamify? An experimental field study of the } \\
\text { influence of badges on motivation, activity, and performance in an } \\
\text { online learning course }\end{array}$ & 2018 & $\begin{array}{l}\text { Kyewski, E.; Kramer, } \\
\text { C. }\end{array}$ & 99th \\
\hline $\mathbf{P 2 8}$ & $\begin{array}{l}\text { On the effectiveness of game-like and social approaches in learning: } \\
\text { comparing educational gaming, gamification \& social networking }\end{array}$ & 2016 & Marcos, L. et al. & 99th \\
\hline
\end{tabular}

\section{RESULTADOS}

A RSL empregada nesta pesquisa avaliou a eficácia e o impacto da gamificação no processo de aprendizagem relatado em cada publicação, classificando os resultados em 'positivo', 'negativo', 'neutro' ou 'positivo e negativo' (Quadro 5). Foi considerado como impacto 'positivo' a pesquisa que obteve alguma vantagem, por exemplo, melhoria no engajamento dos estudantes; 'negativo' quando se verificou alguma desvantagem, por exemplo, diminuição do desempenho dos estudantes; e 'neutro' 
quando não apresentou vantagem ou desvantagem, face ao método tradicional de ensino.

Quadro 5 - Impacto da gamificação na aprendizagem. Fonte: Os autores.

\begin{tabular}{|c|c|}
\hline Impacto & Publicações ID(s) \\
\hline Positivo & P2, P4, P5, P7, P8, P9, P10, P11, P13, P14, P15, P16, P17, P18, P19, P20, P21, P22, P23, \\
\hline Negativo & P24, P25, P26 \\
\hline Neutro & - \\
\hline Positivo e Negativo & P3, P27 \\
\hline
\end{tabular}

A pesquisa identificou os principais benefícios obtidos com a gamificação (Figura 2). O engajamento é o benefício mais frequente nas publicações P1, P4, P6, P7, P9-P12, P16, P17, P21-P23, P25, P26 e P28. Os estudantes que participaram de disciplinas gamificadas sentiram-se mais envolvidos e intergessados com o curso. Os autores das publicações P2, P5, P7, P9-P11, P13-P15, P17, P19, P20, P22-P24 indicaram que a gamificação aumentou a motivação dos estudantes, que passaram, em média, mais tempo realizando tarefas e tentando realizar mais problemas se comparados aos grupos não gamificados. Das 28 publicações, 10 confirmaram que houve melhoria nas notas dos estudantes que participaram das práticas gamificadas. Esses benefícios refletiram um ganho no aprendizado (P7, P10, P14, P15, P17, P18, P22 e P23). As publicações P2, P11, P21 e P22 observaram, via questionários aplicados aos participantes dos cursos gamificados, que os estudantes se sentiram satisfeitos e gostariam de estudar novamente cursos com práticas de gamificação.

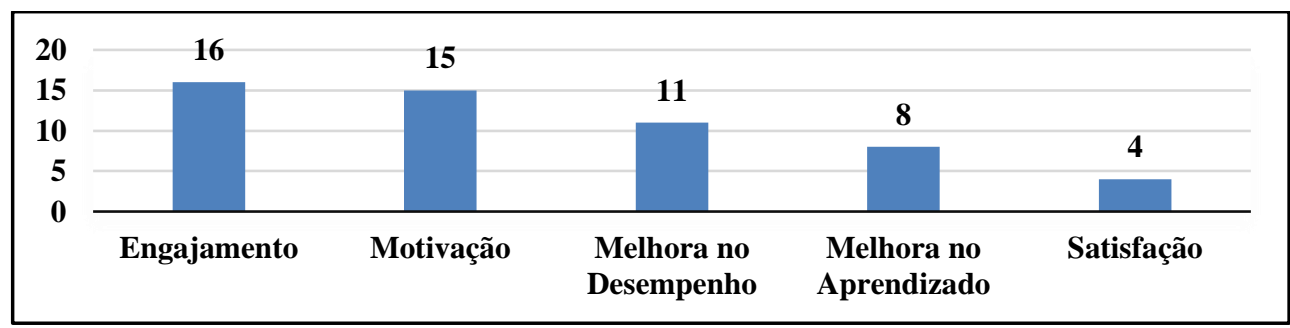

Figura 2 - Principais benefícios obtidos com a gamificação. Fonte: Os autores.

Por fim, observou-se como a gamificação vem sendo aplicada (Figura 3). As publicações P4, P9, P12 e P21 utilizaram elementos de jogos, como pontuação e ranqueamento, em atividades em grupos para o aprendizado. As publicações P3, P5, P8, P15, P19, P23, P26 e P27 utilizaram o Moodle em conjunto com extensões que adicionam elementos de jogos a esta plataforma, tais como experiência, ranqueamentos, avatares e jogos de quiz. As publicações P1, P11, P13, P14, P16, P17, P20, P22, P24, P25 e P28 desenvolveram plataformas e-learning com elementos de gamificação, enquanto P2, P10 e P18 utilizaram jogos sérios para aplicar a gamificação.

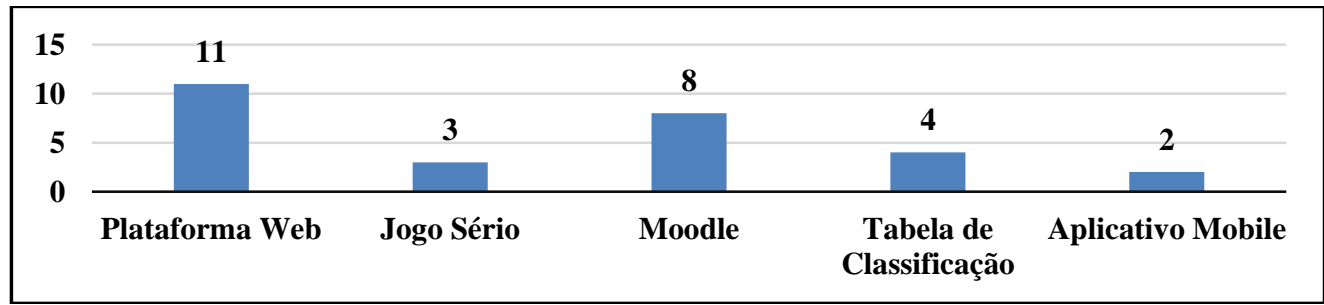

Figura 3 - Formas de uso da gamificação. Fonte: Os autores. 


\section{DISCUSSÃO}

Os resultados e as respostas das questões de pesquisa deste estudo serão discutidos nessa seção.

RQ1. Que evidências existem para a eficácia da gamificação em disciplinas da Computação no ensino superior?

Os experimentos realizados em P2, P4-P5, P7-P11 e P13-P26 mostraram que a gamificação foi eficaz, trazendo diversos benefícios para o processo de aprendizagem. Hardasmal e Salguero (2020) observaram que a média da nota dos estudantes do grupo gamificado foram melhores do que o não gamificado $(7,02 \pm 1,88$ vs. $6,31 \pm 1,62)$. Ademais, a taxa de reprovação foi menor no grupo gamificado, sendo $18,8 \%$ vs. $21,05 \%$ do grupo de controle. Para Pontes et al. (2018) e Maia e Graelml (2015), as experiências alcançaram impactos positivos. No grupo experimental, os estudantes ficaram mais engajados e conseguiram resolver mais problemas difíceis do que o grupo de controle com o método tradicional de ensino. Carreño-León et al. (2018) relataram que os alunos ficaram mais engajados devido à competição vivenciada, mostrando-se satisfeitos com o uso da gamificação. Os experimentos P1, P6, P12 e P28 apresentaram pontos positivos e negativos. Ahmad et al. (2020) observaram que a aplicação em grandes grupos, com sete ou mais estudantes, ao longo do tempo, gerou uma diminuição na motivação dos alunos, enquanto na aplicação em pequenos grupos, ou individualmente, os estudantes tiveram melhores resultados. Portanto, deve-se levar em consideração o tamanho do grupo para melhorar a eficácia do uso da gamificação. Laskowski (2015) notou uma melhora no engajamento no que tange ao processo didático, medido pela presença e pelos trabalhos entregues pelo estudantes do grupo gamificado; contudo, houve uma diminuição da nota se comparada ao grupo não gamificado. Para Watson e Lipford (2019) e Marcos et al. (2016), a gamificação proporcionou melhora no engajamento e/ou na motivação, porém havendo uma diminuição dos benefícios - engajamento e motivação - ao longo do tempo. Em duas publicações não se observou benefícios com a gamificação. García-Iruela et al. (2020) e Kyewski e Kramer (2018) não identificaram diferenças significativas entre os grupos gamificados e não gamificados, não impactando na melhora do desempenho, do engajamento e da motivação.

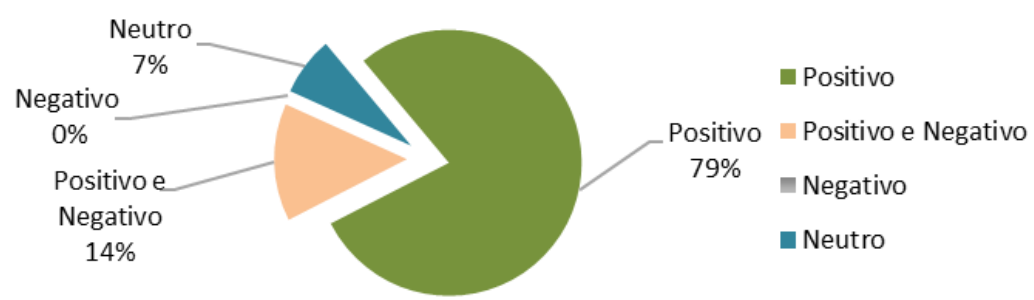

Figura 4 - Impacto da gamificação na aprendizagem. Fonte: Os autores.

Das 28 publicações, 22 delas mostraram evidências de impactos positivos, duas com impacto neutro, quatro positivo e negativo e nenhuma das publicações apresentou somente impacto negativo. A maioria dos estudos demonstrou um impacto positivo do uso da gamificação se comparada ao método tradicional de ensino (Figura 4). Portanto, é importante planejar a forma como os elementos de jogos serão implementados para o processo de aprendizagem, para que se possa obter sucesso no ensino (AHMAD et al., 2020).

RQ2. Quais os beneficios obtidos no processo de aprendizagem com a 
A RSL identificou que os principais benefícios obtidos com a gamificação são engajamento, motivação, melhora no desempenho, no aprendizado e satisfação. $\mathrm{O}$ uso de elementos de gamificação pode ser uma maneira significativa de aumentar, principalmente, a motivação e o engajamento dos estudantes, bem como melhorar a eficácia do aprendizado.

\section{no ensino superior?}

RQ3. Como a gamificação está sendo aplicada em disciplinas da Computação

De acordo com o estudo realizado, verificou-se que a gamificação vem sendo aplicada principalmente com tecnologias de e-learning, tais como o Moodle, plataformas web e aplicativos mobile, incluindo elementos de jogos como pontos, badges de conquistas, níveis de dificuldades e ranqueamentos dos estudantes, para o aprendizado do conteúdo.

\section{CONSIDERAÇÕES FINAIS}

O objetivo principal da RSL empregada nesse estudo foi fornecer uma visão das pesquisas empíricas mais recentes realizadas para comprovar a eficácia do uso da gamificação no ensino e aprendizagem no ensino superior em Ciência da Computação. Os resultados revelaram que a maioria dos experimentos relatou impacto positivo com o uso da gamificação, sendo uma alternativa eficaz para o ensino, trazendo como benefícios melhoria no desempenho, aprendizado, nível de satisfação e, sobretudo, maior engajamento e motivação dos estudantes. Para tanto, a gamificação vem sendo aplicada principalmente por meio do Moodle, aplicações web e aplicativos mobile, utilizando elementos de jogos como pontos, badges, níveis de dificuldades $\mathrm{e}$ ranqueamentos dos estudantes.

Para atingir o objetivo principal do estudo, foi realizada uma RSL a partir da qual foram extraídas e analisadas 151 publicações oriundas de repositórios considerados referências na área. Após a aplicação dos critérios de inclusão e exclusão dos trabalhos foram obtidos 28 artigos relacionados ao objetivo desta pesquisa. Como oportunidades futuras, sugerem-se um maior aprofundamento dos elementos de gamificação mais utilizados e seus reflexos sobre os estudantes, além do estudo sobre os efeitos do uso da gamificação em longo prazo.

\section{REFERÊNCIAS BIBLIOGRÁFICAS}

AHMAD, A. et al. The impact of gamification on learning outcomes of computer science majors. ACM Transactions on Computing Education, v. 20, n. 2, p. 1-25, 2020.

BARTEL, A.; HAGEL, G. Gamifying the learning of design patterns in software engineering education. In: IEEE Global Engineering Education Conference (EDUCON), p. 74-79, 2016.

BERNIK, A. et al. Achievements and usage of learning materials in computer science hybrid courses. Journal of Computer Science, v. 15, n. 3 p. 489-498, 2019.

BERNIK, A. et al. Introducing gamification into e-learning university courses. In: 40th International Convention on Information and Communication Technology, Electronics and Microelectronics, p. 711-716, 2017.

CARRENO-LEON, M. et al. Gamification technique for teaching programming. In: IEEE Global Engineering Education Conference, Tenerife, p. 2009-2014, 2018. 
DICHEVA, D. et al. Gamification in education: a systematic mapping study. Journal of Educational Technology \& Society, v. 18, n. 3, p.75-88, 2015.

ELGRABLY, I. S.; OLIVEIRA, S. R. B. Gamification and evaluation of the use the agile tests in software quality subjects: the application of case studies. In: Proceedings of the 13th International Conference on Evaluation of Novel Approaches to Software Engineering, p. 416-423, 2018.

FACEY-SHAW, L. et al. Digital badges for motivating introductory programmers: qualitative findings from focus groups. In: IEEE Frontiers in Education Conference (FIE), San Jose, CA, p. 1-7, 2018.

FELDBUSCH, L. et al. Smile goes gaming: gamification in a classroom response system for academic teaching. In: Proceedings of the 11th International Conference on Computer Supported Education, p. 268-277, 2019.

FRACZ, W. An empirical study inspecting the benefits of gamification applied to university classes. In: 7th Computer Science and Electronic Engineering Conference, CEEC 2015 Conference Proceedings, p. 135-139, 2015.

GARCÍA-IRUELA, M. et al. Gamification and computer science students activity. IEEE Access, v. 8, p. 96829-96836, 2020.

GIUSEPPINA, P. et al. 2TSW: Automated assessment of computer programming assignments in a gamified web based system. In: 18th International Conference on Information Technology Based Higher Education and Training (ITHET). IEEE, p. 1-9. 2019.

GONZALEZ-ESCRIBANO, A. et al. Toward improving collaborative behaviour during competitive programming assignments. In: IEEE/ACM Workshop on Education for HighPerformance Computing (EduHPC), Denver, CO, USA, p. 68-74, 2019.

HAKULINEN, L. et al. The effect of achievement badges on students' behavior: an empirical study in a university-level computer science course. International Journal of Emerging Technologies in Learning, p. 18-29, 2015.

HARDASMAL, A.; SALGUERO, A. Teaching parallelism with gamification in cellular automaton environments. IEEE Revista Iberoamericana de Tecnologias del Aprendizaje, v. 15, no. 1, p. 34-42, 2020.

IVANOVA, G. et al. Gamification in software engineering education. In: 42nd International Convention on Information and Communication Technology, Electronics and Microelectronics (MIPRO), p. 1445-1450, 2019.

JURGELAITIS, M. et al. Implementing gamification in a university-level UML modeling course: a case study. Computer Applications in Engineering Education, v. 27, n. 2, p. 332$343,2019$.

KAILA, E. et al. A model for gamifying programming education: university-level programming course quantified. In: 41st International Convention on Information and Communication Technology, Electronics and Microelectronics, p. 689-694, 2018.

KITCHENHAM, B. A.; CHARTERS. S. Guidelines for performing systematic literature reviews in software engineering. Technical Report EBSE 2007-001, 2007. 
KUMAR, B.; SHARMA, K. A gamified approach to achieve excellence in programming. In: 4th International Conference on Computing Sciences (ICCS), Jalandhar, p. 107-114, 2018.

KYEWSKI, E.; KRAMER, N. C., To gamify or not to gamify? An experimental field study of the influence of badges on motivation, activity, and performance in an online learning course. Computers \& Education, v. 118, p. 25-37, 2018.

LASKOWSKI, M. Implementing gamification techniques into university study path - a case study. In: IEEE Global Engineering Education Conference, p. 582-586, 2015.

LEHTONEN, T. et al. On the role of gamification and localizattion in na open online learning environment:javala experiences. In: Proceedings of the 15th Koli Calling Conference On Computing Education Research, p.50-59, 2015.

MACENA, J. D. et al. Evaluation of the use of leaderboards in a gamified application for academic learning. RENOTE, v. 18, n. 2, 2020.

MAIA, R. F.; GRAELML, F. R. Playing and learning with gamification: an in-class concurrent and distributed programming activity. In: IEEE Frontiers in Education Conference (FIE), San Jose, CA, USA, p. 1-6, 2015.

MARCOS, L. et al. On the effectiveness of game-like and social approaches in learning: comparing educational gaming, gamification \& social networking. Computers \& Education, v. 95, p. 99-113, 2016.

MARTIN, S. et al. Increasing engagement in a network security management course through gamification. In: IEEE Global Engineering Education Conference (EDUCON), Dubai, United Arab Emirates, p. 1380-1383, 2019.

NAH, H. et al. Gamification of education: a review of literature. In: International Conference on HCI in Business, p. 401-409, LNCS Springer, 2014.

PONTES, R. et al. Analyzing the impact of leaderboards in introductory programming courses' short-length activities. In: IEEE Frontiers in Education Conference (FIE), San Jose, CA, USA, p. 1-9, 2018.

SMIDERLE, R. et al. Studying the impact of gamification on learning and engagement of introverted and extroverted students. In: IEEE 19th International Conference on Advanced Learning Technologies (ICALT), p. 71-75, 2019.

SPENCE, M. et al. Gamifyng a library orientation tutorial for improved motivation and learning. In: 2012 ASEE - American Society for Engineering Education Annual Conference, 2012.

SPRINT, G.; COOK, D. Enhancing the CS1 student experience with gamification, IEEE Integrated STEM Education Conference, Princeton, NJ, p. 94-99, 2015.

TSAY, C. et al. Enhancing student learning experience with technology-mediated gamification: an empirical study. Computers \& Education, v. 121, p. 1-17, 2018.

WATSON, S.; LIPFORD, H. R. Motivating students beyond course requirements with a serious game. In: Proceedings of the 50th ACM Technical Symposium on Computer Science Education, p. 211-217, 2019. 